International Journal of Modern Physics B, (C) World Scientific Publishing Company

\title{
RENORMALIZATION GROUP APPROACHES TO STRONGLY CORRELATED ELECTRON SYSTEMS
}

\author{
A.C. HEWSON and S.C. BRADLEY \\ Mathematics Department, Imperial College, \\ London, $S W^{7} 2 B Z$, United Kingdom \\ R. BULLA \\ Theoretische Physik III, Elektronische Korrelationen und Magnetismus, Institut für Physik, \\ Universität Augsburg, D-86135 Augsburg, Germany \\ Y. $\overline{\mathrm{ONO}}$ \\ Department of Physics, Nagoya University, \\ Nagoya 464-8602, Japan \\ Received (received date) \\ Revised (revised date)
}

\begin{abstract}
In recent years the numerical renormalization group method (NRG) has been extended to the calculation of dynamic response functions and transport properties of magnetic impurity models. The approach can now be applied more widely to lattice models of strongly correlated electron systems by the use of dynamical mean field theory (DMFT), in which the lattice problem is transformed into one for an effective impurity with an additional self-consistency constraint. We review these developments and assess the potential for further applications of this approach.

We also discuss an alternative approach to renormalization, renormalized perturbation theory, in which the leading asymptotically exact results for the low temperature regime for a number of magnetic impurity models can be obtained within finite order perturbation theory.
\end{abstract}

Keywords: Renormalization; strongly correlated electron systems; dynamical mean field theory: PACS $71.27 .+\mathrm{a}, 71.10 . \mathrm{Fd}$ and $75.20 . \mathrm{H}$

\section{Introduction}

Since their successful application to critical phenomena in the early 1970 s, renormalization group methods have been applied to many areas of condensed matter physics. The pioneering work in developing this technique was largely due to Kenneth Wilson and one of the high points of his achievements was the formulation and application of the numerical renormalization group (NRG) ${ }^{1}$ to provide a fully quantitative understanding of the Kondo problem ${ }^{2,3}$. Wilson's calculations were extended to other magnetic impurity models soon afterwards ${ }^{4}$. These were calculations for the thermodynamic behavior of these models and it is only relatively recently that this approach has been extended to enable the dynamic response 
functions and transport properties of magnetic impurities to be calculated ${ }^{5,6}$. The generic model in this context is the single site Anderson impurity model (SIAM) ${ }^{7}$ and our discussion of the NRG approach will be based on this model. It describes localized d or f states, associated with the partially filled $3 \mathrm{~d}$ or $4 \mathrm{f}$ shells of a transition metal or rare earth impurity ion, hybridized with the conduction band states of a host metal,

$$
H=\sum_{\sigma} \varepsilon_{f} f_{\sigma}^{\dagger} f_{\sigma}+U f_{\uparrow}^{\dagger} f_{\uparrow} f_{\downarrow}^{\dagger} f_{\downarrow}+\sum_{\mathbf{k}, \sigma}\left(V_{\mathbf{k}} f_{\sigma}^{\dagger} c_{\mathbf{k} \sigma}+V_{\mathbf{k}}^{*} c_{\mathbf{k} \sigma}^{\dagger} f_{\sigma}\right)+\sum_{\mathbf{k}, \sigma} \varepsilon_{\mathbf{k} \sigma} c_{\mathbf{k} \sigma}^{\dagger} c_{\mathbf{k} \sigma},
$$

where $\varepsilon_{f}$ is the energy of the localized state, $\varepsilon_{\mathbf{k}}$ is the energy of the Bloch states of the host metal and $V_{\mathbf{k}}$ the hybridization matrix element between the localized state and the conduction electrons. The localized state is taken to be non-degenerate but can be occupied by a spin up or spin down electron, or one of each. When this state is occupied by two electrons there is a large Coulomb interaction between them and this term is included in the Hamiltonian with a matrix element $U$. In the "atomic limit' $V_{\mathbf{k}}=0$, there is a local moment regime when $\varepsilon_{f}$ lies below the Fermi-level of the host metal and is occupied by a single electron ( $\uparrow$ or $\downarrow$ ), and the energy level $\varepsilon_{f}+U$ for a second electron is empty as it lies above the Fermi-level. When weak hybridization is included, the model in this regime can be mapped into a Kondo model of a localized spin interacting via an antiferromagnetic exchange interaction with the spins of the conduction electrons.

The one-electron Green's function for the localized f-electron $G_{\sigma}^{(0)}(\omega)$ in the non-interacting limit $U=0$ takes the form,

$$
G_{\sigma}^{(0)}(\omega)=\frac{1}{\omega-\varepsilon_{f}-\Delta(\omega)}
$$

where $\Delta(\omega)=\sum_{\mathbf{k}}\left|V_{\mathbf{k}}\right|^{2} /\left(\omega-\varepsilon_{\mathbf{k}}\right)$. In the case of a wide flat conduction band, $\Delta(\omega+i \delta)=-i \Delta$, and the spectral density corresponding to this Green's function $\rho^{0}(\omega)$ takes the form of a Lorentzian resonance centered at $\varepsilon_{f}$

$$
\rho^{0}(\omega)=\frac{\Delta / \pi}{\left(\omega-\varepsilon_{f}\right)^{2}+\Delta^{2}} .
$$

When the interaction term is taken into account and a self-energy term $\Sigma(\omega)$ is included the local Green's function takes the form,

$$
G_{\sigma}(\omega)=\frac{1}{\omega-\varepsilon_{f}-\Delta(\omega)-\Sigma(\omega)} .
$$

The basic idea of the Wilson style renormalization group method is to eliminate the degrees of freedom associated with the higher energy excitations by taking the virtual interactions they induce into account and projecting onto an effective model for the lower energy states. This is done progressively in a sequence of steps to reach the very low energy states which lie within the thermal energy range. If these effective models are appropriately rescaled then a meaningful comparison can 
be made between the effective models at consecutive steps. It is expected that in a model which has continuous excitations down to the ground state that the renormalization group transformation relating consecutive effective models should have a stable fixed point in the low energy limit. The effective model in this limit with its leading correction terms determines the low temperature thermodynamics of the system.

To set up such a procedure for impurity models, such as the Anderson model, Wilson divided the interval of the conduction band, $-D<\epsilon<D$, into a sequence of sub-intervals. The logarithmic integrations that arise in the Kondo problem indicate that all energy scales contribute to the low energy behavior, so the subintervals were chosen such that each of the intervals give equal contributions to such logarithmic integration,

$$
\Lambda^{-n-1}<\epsilon<\Lambda^{-n} \quad \text { or } \quad-\Lambda^{-n}<\epsilon<-\Lambda^{-n-1} . \quad n=0,1,2 \ldots
$$

Within each sub-interval, only one state was retained, the one directly hybridized with the impurity, so that the continuous spectrum of conduction states is approximated by a discrete which has limit point at the Fermi-level. The parameter $\Lambda>1$ controls the discretization and in practice is chosen to be in the range $1.5<\Lambda<3$. These conduction states are then transformed into a tight-binding chain with the states of the impurity coupled at one end. With these transformations the Anderson model takes the form,

$H=\sum_{\sigma} \varepsilon_{f} f_{\sigma}^{\dagger} f_{\sigma}+U f_{\uparrow}^{\dagger} f_{\uparrow} f_{\downarrow}^{\dagger} f_{\downarrow}+V \sum_{\sigma}\left(f_{\sigma}^{\dagger} c_{0 \sigma}+c_{0 \sigma}^{\dagger} f_{\sigma}\right)+\sum_{i=0, \sigma}^{i=\infty}\left(t_{i, i+1} c_{i \sigma}^{\dagger} c_{i+1 \sigma}+\right.$ h.c. $)$,

where the hopping matrix elements decrease exponentially along the chain and $\lim _{i \rightarrow \infty} t_{i, i+1}=0$.

With the Hamiltonian in the form (6) a renormalization group procedure, which takes account of excitations on a sequence of reducing energy scales, can be set up. Starting at the impurity site the Hamiltonian for a finite section of the chain, as indicated in figure 1, is diagonalized numerically and all the many-body states and energy levels are calculated. The length of the chain is increased in the next step by including the next site along the chain, and the states of this longer chain are again calculated numerically. These steps are repeated to explore lower and lower energy scales of the system. If all the states could be retained at each stage there would be no point in this iterative procedure but, when the chain length has about six of seven sites, the matrices become too large to retain all the states, so only the lowest 500-1000 states are retained in subsequent steps. It was demonstrated by Wilson in his calculations for the Kondo problem ${ }^{1}$ that, with a suitable choice for $\Lambda$, this is quite sufficient to be able to obtain accurate predictions for the low energy behavior of the model. 


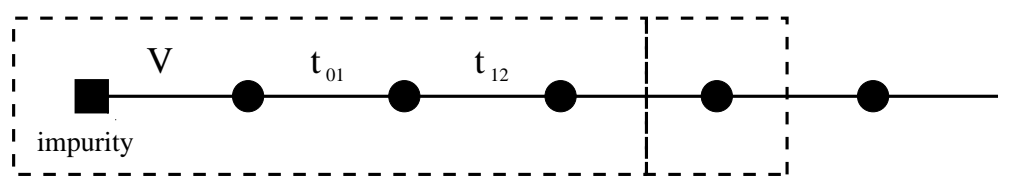

Figure 1. The linear chain form of the Anderson model corresponding to the Hamiltonian $(6)$.

In extending the approach to the calculation of dynamic properties of the impurity, such as the spectral density of the localized electrons $\rho_{\sigma}(\omega)$, matrix elements of the local operators have also to be calculated iteratively. The spectral density at a particular energy scale $\omega$ is evaluated directly from the definition,

$$
\rho_{\sigma}(\omega)=\frac{1}{Z} \sum_{m, n}\left|\left\langle n\left|f_{\sigma}^{\dagger}\right| m\right\rangle\right|^{2} \delta\left(\omega-E_{n}+E_{m}\right)\left(e^{-\beta E_{m}}+e^{-\beta E_{n}}\right),
$$

using the results from the diagonalization of the chain appropriate to this energy scale, where $|n\rangle$ is a many-body eigenstate with a corresponding energy level $E_{n}, Z$ is the partition function, and $\beta=1 / T$, where $T$ is the temperature. Due to the use of a discrete set of states for the conduction states, the calculated spectral density consists of a set of delta functions, which are then broadened on a logarithmic scale to give a continuous spectrum. Most of the calculations for the dynamics have been for $T=0$, and give a very precise resolution of the spectra on the very low energy scales. Sum rules, such as the requirement that the integral of the spectral density $\rho_{\sigma}(\omega)$ should be unity, are not automatically satisfied and provide some estimate of the level of approximation involved in these calculations: they are usually satisfied to within a few percent. Calculations of the dynamics at finite temperatures have also been performed ${ }^{6}$. In this case there are two energy scales, that corresponding to $\omega$ and that corresponding to $T$, and the results used in the evaluation of (7) are taken to be those on an energy scale corresponding to whichever is the largest of $\omega$ and $T$.

These types of calculations have now been applied to the calculation of thermodynamics, dynamics and transport properties of a range of impurity models which include degeneracy ${ }^{5}$, magnetic field ${ }^{8}$, crystal fields ${ }^{9}$, models in which the conduction electrons are superconducting ${ }^{10}$, or have a pseudogap at the Fermi-level ${ }^{11,12}$, two-channel Kondo models ${ }^{13,14}$, and an impurity model with a low energy marginal fixed point ${ }^{15,14}$. Some recent applications have been to mesoscopic systems and quantum dots ${ }^{16,17,18}$. In applications to mesoscopic systems it is possible to change the parameter regime of the model by changing a gate voltage. This should make possible a more detailed comparison between theory and experiment than is possible for a magnetic impurity system, where the parameters cannot easily be modified in a controlled way. The approach has also been applied to two level systems, to resolve some controversial problems regarding the behavior in the intermediate regime 
when the quantum tunnelling becomes suppressed by dissipation ${ }^{19}$, and to models with anisotropic interactions ${ }^{20}$.

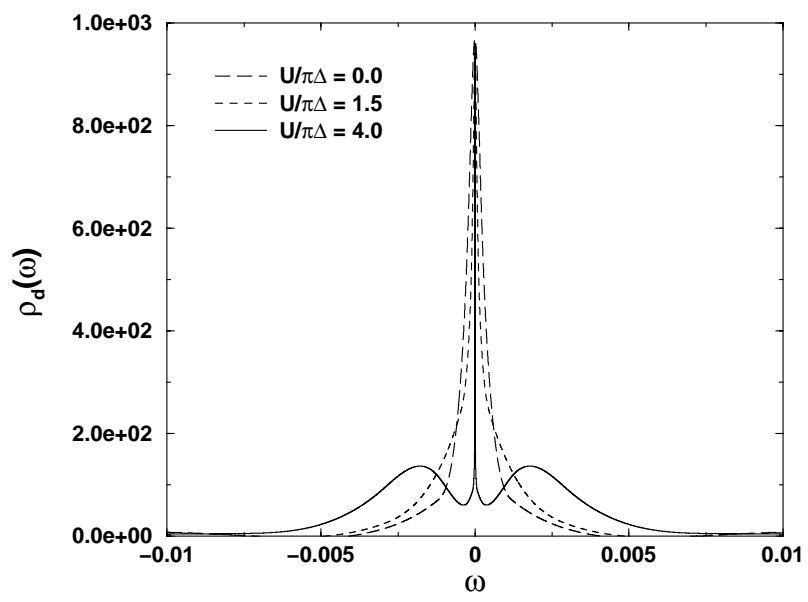

Figure 2. The spectral density $\rho_{d}(\omega)$ of the local Green's function for the SIAM with $V=0.01414\left(\Delta=10^{-4} \pi\right)$.

To illustrate the results we show in figure 2 the spectral density $\rho(\omega)$ of the local Green's function for the particle-hole symmetric Anderson model for a range of values of the interaction $U$. The peak at the Fermi-level for $U=0$ corresponds to the virtual bound state resonance given by equation (3). As $U$ is increased new peaks appear at $\omega= \pm U / 2$, corresponding to the broadened 'atomic' levels. The very narrow resonance seen at the Fermi-level in the large $U$ regime is the Kondo resonance, and has a width of the order of the Kondo temperature $T_{\mathrm{K}} \sim$ $U e^{-U / \pi \Delta}$. It reflects the residual scattering from the impurity surrounded by a cloud of conduction electrons which screen out the impurity magnetic moment such that the ground state of the system is a singlet with a finite magnetic susceptibility at $T=0$. This low temperature fixed point corresponds to a Fermi-liquid, but one in which the power-laws $\left(T / T_{\mathrm{K}}\right)^{n}$, (integer $n$ ), have enhanced coefficients due to the narrowness of the Kondo peak $\left(T_{\mathrm{K}}\right)$.

We contrast this case with the results for the $\mathrm{O}(3)$ Anderson model, which corresponds to the particle-hole symmetric Anderson model expressed in terms of four Majorana fermions, with one of the local Majorana modes uncoupled from the conduction electrons ${ }^{14,21}$. In the Kondo or large $U$ limit it can be mapped into the Hamiltonian which describes the spin excitations of the linear dispersion two channel Kondo model. The $\mathrm{O}(3)$ model has a marginal Fermi-liquid fixed point due to scattering with the singular unhybridized Majorana mode, and the linear $\omega$ dependence of the spectral densities of the Majorana fermions in the vicinity of the Fermi-level can be clearly seen in the NRG results shown in figure 3 . 


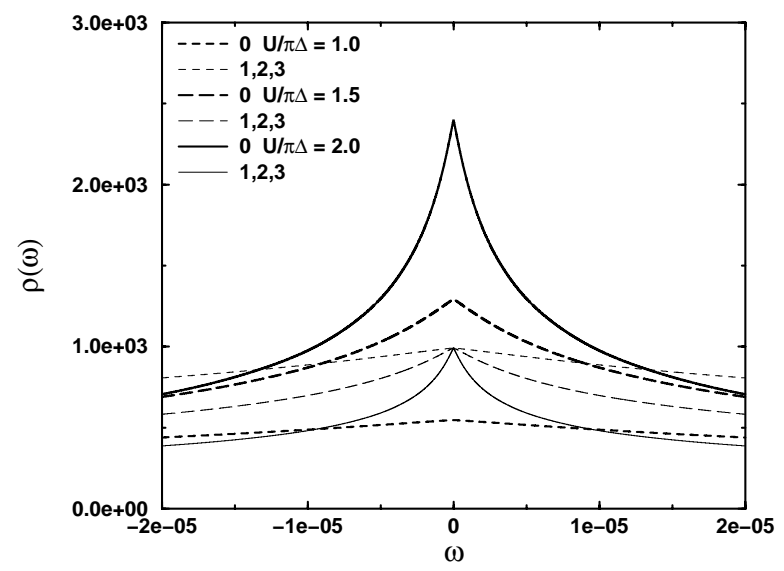

Figure 3. The spectral density $\rho(\omega)$ of the Majorana fermion impurity Green's function in the marginal Fermi liquid situation with the hybridization, $V=0.01414$ for all the local Majorana modes except the uncoupled one for which $V=0$; taken from reference ${ }^{14}$

The success of the application of the numerical renormalization group to Anderson, and other impurity models, in the large $U$ or strong correlation regime, leads naturally to the question as to whether the approach can be developed or extended to deal with lattice models, such as the Hubbard model, in which there is a local interaction $U$ at every lattice point. The Hamiltonian for the Hubbard model is

$$
H=-\sum_{<i, j>, \sigma} t_{i, j}\left(c_{i \sigma}^{\dagger} c_{j \sigma}+\text { h.c. }\right)+U \sum_{i} c_{i \uparrow}^{\dagger} c_{i \uparrow} c_{i \downarrow}^{\dagger} c_{i \downarrow},
$$

which describes conduction electrons hopping between orbitals located at lattice sites and a local Coulomb interaction $U$. In the one dimensional case, with nearest neighbour hopping, the similarity of this model with the linear chain form for the Anderson model, as used in the NRG calculation (6), would suggest that the NRG technique could be applied directly to this situation. However, the similarity is deceptive; the hopping matrix elements, $t_{i, i+1}$ of the impurity model decrease with $i$, and this form is possible because it is a calculation purely for the impurity contribution. For a real one-dimensional lattice with translational invariance the nearest neighbour hopping term will be independent of the site index. As a result serious problems arise in the NRG when the higher energy states are discarded in working along the chain as there is a complete mismatch between the energy scale of the states retained, which decreases with each site that is added, and the scale of the hopping matrix element to the extra site which remains the same. The impurity problem can be set up such that these energy scales decrease in parallel. The successful generalization of the numerical renormalization group technique to one-dimensional systems has proved to be a difficult problem but Steve White has devised a modified form such that the problem of the boundary condition with the 
extra site is overcome by adding the new sites to the centre of the chain ${ }^{22}$. A preliminary calculation is then carried out to calculate a reduced density matrix for the states which are retained for the subsequent steps. This approach, the density matrix renormalization group (DMRG), has proved to be a very effective way of calculating the ground state and low energy excitations for many one-dimensional models, and has been applied widely. Based on the transfer matrix approach, the method has also been extended to the calculation of the thermodynamic and dynamical response functions for some one-dimensional models. The technique, though similar in its philosophy, is quite a different in practice from the NRG as applied to impurity models. Details of the DMRG approach and its application can be found in reference ${ }^{23}$. There is, however, a direct way of applying the impurity NRG to lattice models based on dynamical mean field theory (DMFT) ${ }^{24}$, which we describe briefly in the next section.

\section{Dynamical Mean Field Theory}

In dynamical mean field theory the lattice problem is mapped into an impurity one with an additional constraint. This is only possible if the interaction terms are on-site and the self-energy is local so that it depends on the frequency $\omega$ only, independent of the wavevector $\mathbf{k}$. The first condition is satisfied for the Hubbard model (8), but the second condition is not, in general, but can be satisfied for certain lattice models in the limit of infinite dimensionality ${ }^{25}$, if the hopping matrix elements are scaled appropriately with the dimensionality $d$. If one particular site is regarded as an impurity, the effective Lagrangian obtained by formally integrating out the states on other sites, has the same form as that for an impurity and the electron self-energy $\Sigma(\omega)$ is given by the same set of diagrams as for the impurity. The propagator in these diagrams, the non-interacting Green's function $\mathcal{G}_{0}(\omega)$ for the effective impurity, is not known as it depends on how the electron propagates when it leaves the impurity site and interacts with the electrons on the other sites. However, there is nothing special about the site which is singled out to be regarded as an impurity, and so using this fact, a self-consistency condition can be derived which is sufficient for its determination. This self-consistency condition is typical of mean field theories, but it is usually a time-independent field and not, as here, a dynamic function that is determined self-consistently. For a Bethe lattice with a nearest neighbour hopping matrix element $t / \sqrt{d}$ the self-consistency condition is

$$
\mathcal{G}_{0}(\omega)^{-1}=\omega+\mu-t^{2} G(\omega),
$$

in the limit $d \rightarrow \infty$. The Green's function $\mathcal{G}_{0}(\omega)$ for the effective impurity can be taken to be of the form (2) for the Anderson model, with the function $\Delta(\omega)$ to be determined from the self-consistency condition. The self-consistency equation (9) then simplifies to

$$
\Delta(\omega)=t^{2} G(\omega) .
$$

The non-interacting Green's function for the effective impurity $\mathcal{G}_{0}(\omega)$ should not be confused with the non-interacting Green's function for real lattice $G_{0}(\mathbf{k}, \omega)$ which 
has a $\mathbf{k}$ dependence. The non-interacting density of states $D_{0}(\omega)$ corresponding to k-integrated Green's function $G_{0}(\omega)$, for a Bethe lattice is given by

$$
D_{0}(\omega)=\frac{1}{\pi t} \sqrt{1-\left(\frac{\omega}{2 t}\right)^{2}} .
$$

In the DMFT the density of states for the interacting model, $D(\omega)$, is given by $D(\omega)=D_{0}(\omega-\Sigma(\omega))$.

The DMFT approach is only approximate for systems with a finite dimensionality $d$. There is experimental evidence, however, in strongly correlated systems in three dimensions, such as in heavy fermion materials, that the $\omega$ dependence of the self-energy is much greater than the $\mathbf{k}$ dependence, so that this approximation should be a good starting point for a theory of these materials.

To realize the DMFT scheme in practice some method is required for calculating the self-energy $\Sigma(\omega)$ of the effective impurity, and many techniques have been used, quantum Monte Carlo ${ }^{26}$, iterated second order perturbation theory (IPT) ${ }^{27}$, exact diagonalization (ED) ${ }^{28}$, and the numerical renormalization group (NRG) ${ }^{29,30,31}$. The quantum Monte Carlo method is the one that has been used most extensively but, due to the restriction on the number of mesh points that can be used in practice for the imaginary time parameter $\tau, 0<\tau<\beta$, it cannot be extended to arbitrarily low temperatures. The NRG approach has two distinct advantages, it can resolve very low energy scales and is applicable at $T=0$. For this reason it has been particularly useful in clarifying the nature of the Mott metal-insulator transition in the single band Hubbard model, and whether, or not, there is a hysterisis region at very low temperatures. In IPT at $T=0$ two co-existing mean field solutions ${ }^{27}$ were found for $U$ in a range $U_{c 1}<U<U_{c 2}$. The second order perturbation theory on which the IPT is based is known to work well for the particle-hole Anderson impurity model at moderately large values of $U$ and a constant $\Delta$, but there is some uncertainty about its applicability in the DMFT because the self-consistency condition imposes a strong $\omega$-dependence on the $\Delta(\omega)$ for the effective impurity. The Monte Carlo method, on the other hand, is a well-controlled approximation though not applicable at very low temperatures. The lowest temperature Monte Carlo results $^{32}$ showed no evidence of a hysterisis region, and the compatibility of the iterated second order perturbation (IPT) results at $T=0$ with some Fermiliquid relations was also questioned ${ }^{33}$. The picture that has emerged from the $T=0$ NRG calculations ${ }^{29,30,31}$ is qualitatively similar to that of the second order iterated perturbation theory (IPT), and has two co-existing solutions, though the range of $U$ over which this occurs is somewhat smaller than that found using IPT. The actual metal-insulator transition at $T=0$ to a paramagnetic state for a Bethe lattice, with antiferromagnetism arising from kinetic exchange frustrated, takes place at $U_{\mathrm{c} 2}$ (the metallic solution when it exists always gives the lowest energy at $T=0$ ), at a value $U_{\mathrm{c} 2}=5.88 t^{31}$, which is lower than the IPT result, $U_{\mathrm{c} 2}=6.6 t^{27}$. The narrowing of the quasi-particle peak with increasing $U$, and its eventual disappearence at a critical value $U_{c}$, and the finite gap for $U>U_{c}$ can be seen clearly in the NRG results 
shown in figure 4 . for the case of a Bethe lattice. Further NRG results for the Mott transition at $T=0$ can be found in reference ${ }^{31}$. Recently NRG results ${ }^{34}$ have been extended to a temperature range which overlaps with recent Monte Carlo results, and the results of the two methods are in complete agreement; the revised Monte Carlo calculations now also see evidence of hysterisis below a critical temperature $T_{\mathrm{c}}{ }^{35}$.

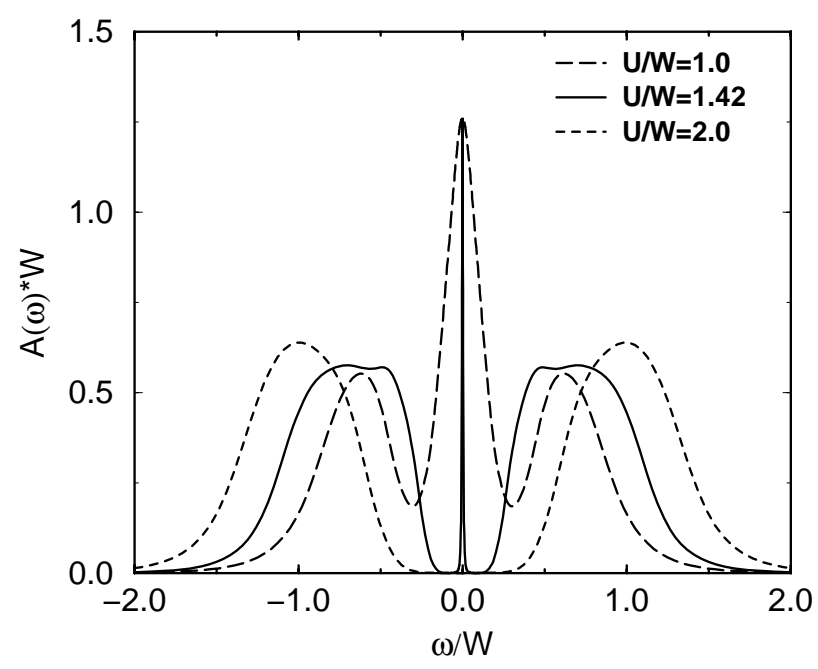

Figure 4. Spectral functions for the Hubbard model on a Bethe lattice for various values of $U$. A narrow quasi-particle peak develops at the Fermi level which vanishes at the critical $U_{\mathrm{c}} \approx 1.47 \mathrm{~W}$, where the bandwidth $W=4 t$

Very close to the Mott transition the quasi-particle peak becomes so narrow that it becomes reasonable to approximate it by a delta-function. In this almost localized limit, Bulla ${ }^{36}$ has shown that a good estimate of the critical value $U_{c 2}$ can be obtained from the zero band-width limit, which corresponds to the first step of the NRG calculation. The solution is required for this two-site problem in the weak hybridization limit, which can be handled analytically. The approximation gives a value of $U_{c 2}=6.0 t$, very close to the estimate from the full NRG calculation $U_{c 2}=5.88 t$. This approach, which has been termed a 'linearized dynamical mean field theory', has been extended to the d-p model ${ }^{37}$,

$$
\begin{gathered}
H=\frac{t_{p d}}{\sqrt{d}} \sum_{<i, j>, \sigma}\left(d_{i \sigma}^{\dagger} p_{j \sigma}+\text { h.c. }\right)+U \sum_{i} d_{i \uparrow}^{\dagger} d_{i \uparrow} d_{i \downarrow}^{\dagger} d_{i \downarrow}+ \\
\frac{t_{d d}}{d} \sum_{i, i^{\prime}, \sigma}\left(d_{i \sigma}^{\dagger} d_{i^{\prime} \sigma}+\text { h.c. }\right)+\varepsilon_{d} \sum_{i, \sigma} d_{i \sigma}^{\dagger} d_{i \sigma}+\frac{t_{p p}}{d} \sum_{j, j^{\prime}, \sigma}\left(p_{j \sigma}^{\dagger} p_{j^{\prime} \sigma}+\text { h.c. }\right)+\varepsilon_{p} \sum_{j, \sigma} p_{j \sigma}^{\dagger} p_{j \sigma}
\end{gathered}
$$

This model is essentially a two-band Hubbard type model with an on-site interaction $U$ in a d-band, which is hybridized with a non-interacting p-band. It has been 
used to describe the copper d-holes and oxygen p-holes in the $\mathrm{CuO}_{2}$ planes of the cuprate superconductors. The phase diagram for the metal-insulator transition is much more complex in this case than in the single band Hubbard model, as there are many more parameters $t_{d d}, t_{p p} t_{p d}, \varepsilon_{p}-\varepsilon_{d}, U$ and the chemical potential $\mu$. In different parameter regimes the insulating state varies from a Mott-Hubbard type insulator, as shown in figure 4 for the single band case, to a charge-transfer type insulator where a gap develops between lower Hubbard band and the p-band.

The two-site calculations of the 'linearized dynamical mean field theory' are analytically tractable in the weak hybridization limit and equations can be derived that generate the complete metal-insulator phase diagram ${ }^{37}$. An example, taken from some of the results, is shown in figure 5. A comparison is made with results of the exact diagonalization method (ED). The ED method gives similar estimates for $U_{c 2}$ for $t_{d d}=0$ to those obtained using the NRG approach for the one-band Hubbard model. The comparison indicates that the linearized DMFT results are accurate to within $5 \%$.

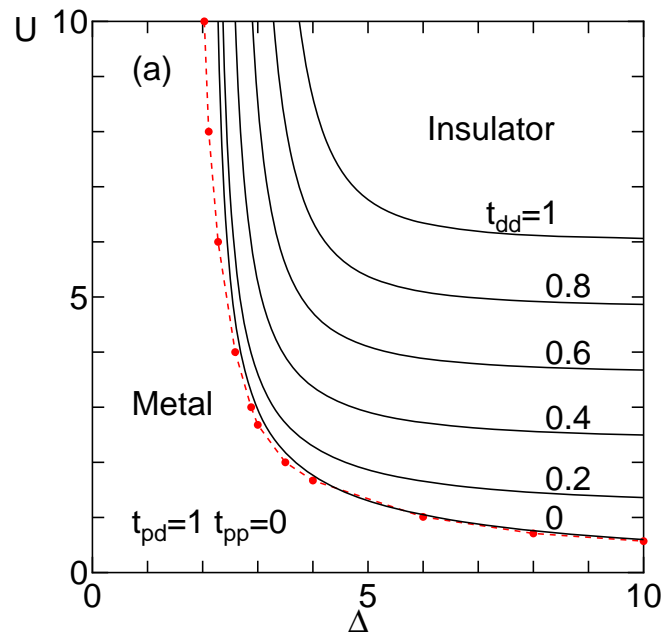

Figure 5. The metal-insulator phase diagram for the $\mathrm{d}-\mathrm{p}$ model for $t_{p p}=0$ and various values of $t_{d d}$ using the linearized DMFT approach, taken from reference ${ }^{37}$. The points which are linked by the dotted curve are the results of DMFT calculations using the ED method.

The full NRG approach within DMFT has also been applied to calculations for the infinite dimensional periodic Anderson model ${ }^{38}$, to study the physics of heavy fermion systems. It is currently being extended to the Holstein-Hubbard Model, which is a generalization of the Hubbard model in which there is a coupling to the lattice via local Einstein phonons of frequency $\omega_{0}$,

$$
H=-\sum_{<i, j>, \sigma} t_{i, j}\left(c_{i \sigma}^{\dagger} c_{j \sigma}+\text { h.c. }\right)+U \sum_{i} c_{i \uparrow}^{\dagger} c_{i \uparrow} c_{i \downarrow}^{\dagger} c_{i \downarrow}
$$




$$
+\lambda \sum_{i}\left(\sum_{\sigma} n_{i, \sigma}-1\right)\left(b_{i}^{\dagger}+b_{i}\right)+\sum_{i} \omega_{0} b_{i}^{\dagger} b_{i} .
$$

For $U=0$ the model corresponds to the Holstein model which has been used extensively to study small polaron behavior, but usually with one or two electrons only in the system. Calculations for this model using NRG and DMFT should enable one to study polaronic motion when the band has finite filling. With $U \neq 0$ the interplay of lattice coupling and strong correlation can be investigated. It should also be possible to perform calculations with an added Hund's rule exchange coupling to a localized spin, which would be a fully quantum mechanical model applicable to the study of the behavior of manganites and the phenomenon of colossal magnetoresistance in these materials.

\section{Other Approaches to Renormalization}

The Wilson style renormalization techniques, in which higher energy excitations are removed progressively to obtain a renormalized Hamiltonian for the low energy scales or, as in the calculation of critical exponents, where the short range fluctuations are integrated out to obtain an effective model for the long wavelength fluctuations, are the ones which are most commonly used in condensed matter physics. The original approach to renormalization, as developed to eliminate the divergences due to a lack of an ultra-violet cut-off in quantum electrodynamics, is not used in condensed matter theory as these type of divergences do not occur in condensed matter problems, so there seems to be no reason to invoke this technique. However, this approach is simply a reorganization of perturbation theory such that the perturbation expansion is in powers of the effective interaction between fully-dressed particles. This approach could be appropriate for strongly correlated systems in condensed matter where there are very large renormalizations of the mass and the interactions, such as in heavy fermion systems. It could be useful to formulate the perturbation theory in terms of the fully dressed quasi-particles and their interactions for calculations of the behavior in the low temperature regime. We illustrate here how this technique can be applied to the Anderson model. Quite remarkably, the exact low temperature behavior of this model can be found by working only up to second order in terms of the renormalized interaction. We also make connections between this approach and the Wilson style calculations.

First of all, we briefly consider how this approach is used in field theory and then apply an equivalent procedure to the Anderson model. In the field theory ${ }^{39}$, such as the $\phi^{4}$ theory, we start with the Lagrangian of the system $\mathcal{L}(\phi, m, g)$ in terms of the field $\phi$, the mass $m$ of the particles and the interaction strength $g$. We choose to absorb all the mass renormalization from the beginning and work with the final or renormalized mass $\tilde{m}$, and also the final or renormalized interaction strength $\tilde{g}$ and the fully renormalized fields $\tilde{\phi}$. We then rewrite the Lagrangian in the form,

$$
\mathcal{L}(\phi, m, g)=\mathcal{L}(\tilde{\phi}, \tilde{m}, \tilde{g})+\mathcal{L}^{\text {counter }}\left(\lambda_{1}, \lambda_{2}, \lambda_{3}\right),
$$

where the terms left over are known as the counter terms, which depend upon the 
differences between the renormalized and bare parameters, but can be alternatively be written in terms of three unknown parameters $\lambda_{1}, \lambda_{2}, \lambda_{3}$. The expansion is carried out in powers of $\tilde{g}$ and the unknown counter terms, $\lambda_{1}, \lambda_{2}, \lambda_{3}$; these latter are determined by the condition that they must cancel out the renormalizations, to the mass, interaction strength and field, as we are working with the fully renormalized parameters. To carry out the expansion we start with the Green's function for the bare fields,

$$
G\left(\mathbf{k}^{2}\right)=\frac{1}{m+\mathbf{k}^{2}+\Pi\left(\mathbf{k}^{2}\right)}
$$

where $\Pi\left(\mathbf{k}^{2}\right)$ is the self-energy term, and separate out $\Pi(0)$ and $\Pi^{\prime}(0)$,

$$
\Pi\left(\mathbf{k}^{2}\right)=\Pi(0)+\mathbf{k}^{2} \Pi^{\prime}(0)+\Pi^{\mathrm{rem}}\left(\mathbf{k}^{2}\right)
$$

and substitute this back into (15) to give

$$
G\left(\mathbf{k}^{2}\right)=\frac{z}{\tilde{m}+\mathbf{k}^{2}+\tilde{\Pi}\left(\mathbf{k}^{2}\right)},
$$

where

$$
\tilde{m}=z(m+\Pi(0)), \quad \tilde{\Pi}\left(\mathbf{k}^{2}\right)=z \Pi^{\mathrm{rem}}\left(\mathbf{k}^{\mathbf{2}}\right), \quad z=1 /\left(1-\Pi^{\prime}(0)\right) .
$$

The fields can be rescaled to eliminate the wavefunction renormalization factor $z$ so that the Green's function for the renormalized fields becomes

$$
\tilde{G}\left(\mathbf{k}^{2}\right)=\frac{1}{\tilde{m}+\mathbf{k}^{2}+\tilde{\Pi}\left(\mathbf{k}^{2}\right)},
$$

where $\tilde{\Pi}\left(\mathbf{k}^{\mathbf{2}}\right) \sim \mathbf{k}^{\mathbf{4}}$.

This Green's function for $\tilde{\Pi}=0$ is taken to be the propagator for the "noninteracting' renormalized particles. The expansion is carried out in powers of the effective interaction between the renormalized particles which is taken to be the renormalized four vertex $\tilde{\Gamma}\left(\mathbf{k}_{\mathbf{1}}, \mathbf{k}_{\mathbf{2}}, \mathbf{k}_{\mathbf{3}}, \mathbf{k}_{\mathbf{4}}\right)$ at a specific point, which we take to be $\mathbf{k}_{\mathbf{1}}=\mathbf{k}_{\mathbf{2}}=\mathbf{k}_{\mathbf{3}}=\mathbf{k}_{\mathbf{4}}=0$. The perturbation expansion is carried out in powers of $\tilde{g}$; it is assumed that the counter-terms $\lambda_{1}, \lambda_{2}, \lambda_{3}$ can also be expressed in powers of $\tilde{g}$ so that they can be determined order by order using the conditions,

$$
\text { (i) } \tilde{\Pi}(0)=0, \quad(\text { ii }) \quad \tilde{\Pi}^{\prime}(0)=0, \quad(\text { iii }) \quad \tilde{\Gamma}(0,0,0,0)=\tilde{g},
$$

which express the fact that the $\tilde{m}, \tilde{g}$ and $\tilde{\phi}$ are fully renormalized. This procedure makes no direct reference to the problems of divegences, and is quite generally applicable. In the field theory context the important question, in the renormalized expansion where $\tilde{m}, \tilde{g}$ and $\tilde{\phi}$ are assumed to be finite, is whether or not there are any divergences remaining due to the lack of an ultraviolet cut-off. If there are no such divergences in the renormalized expansion then the field theory is renormalizable, and the renormalized perturbation expansion can be used to make physical 
predictions. For problems which have a natural ultra-violet cut-off, such as in condensed matter, renormalizability is not an issue and the only question is whether or not there is anything to be gained by reorganising the expansion in this way. We can make a direct connection between this approach in condensed matter and Fermi-liquid theory.

We give an outline of the equivalent procedure ${ }^{40}$ for the Anderson Model. The corresponding Green's function is the local one,

$$
G(\omega)=\frac{1}{\omega-\varepsilon_{f}+i \Delta-\Sigma(\omega)} .
$$

We choose to take into account $\Sigma(0)$ and $\Sigma^{\prime}(0)$ from the beginning, as in the field theory example, using

$$
\Sigma(\omega)=\Sigma(0)+\omega \Sigma^{\prime}(0)+\Sigma^{\mathrm{rem}}(\omega)
$$

so that (21) can be written in the form,

$$
G(\omega)=\frac{z}{\omega-\tilde{\varepsilon}_{f}+i \tilde{\Delta}+\tilde{\Sigma}(\omega)},
$$

where

$$
\tilde{\varepsilon}_{f}=z\left(\varepsilon_{f}+\Sigma(0)\right), \quad \tilde{\Delta}=z \Delta, \quad \tilde{\Sigma}(\omega)=z \Sigma^{\mathrm{rem}}(\omega),
$$

where have used the Luttinger result $\operatorname{Im} \Sigma^{\prime}(0)=0$, and $z=1 /\left(1-\Sigma^{\prime}(0)\right)$.

The fermion fields are rescaled so that

$$
\tilde{G}(\omega)=\frac{1}{\omega-\tilde{\varepsilon}_{f}+i \tilde{\Delta}-\tilde{\Sigma}(\omega)} .
$$

For $\tilde{\Sigma}=0$ this Green's function describes quasi-particles with a renormalized resonance at $\tilde{\varepsilon}_{f}$ of width $\tilde{\Delta}$, with a spectral density,

$$
\tilde{\rho}_{0}(\omega)=\frac{\tilde{\Delta} / \pi}{\left(\omega-\tilde{\varepsilon}_{f}\right)^{2}+\tilde{\Delta}^{2}} .
$$

The renormalized interaction $\tilde{U}$ between these quasi-particles we take to be the four-vertex $z^{2} \Gamma_{\uparrow, \downarrow}\left(\omega_{1}, \omega_{2}, \omega_{3}, \omega_{4}\right)$ evaluated at $\omega_{1}=\omega_{2}=\omega_{3}=\omega_{4}=0$, in analogy with the field theory example. The Lagranian for the Anderson model can now be written in a similar form to (14),

$$
\mathcal{L}\left(\varepsilon_{f}, \Delta, U\right)=\mathcal{L}\left(\tilde{\varepsilon}_{f}, \tilde{\Delta}, \tilde{U}\right)+\mathcal{L}^{\text {counter }}\left(\lambda_{1}, \lambda_{2}, \lambda_{3}\right) .
$$

The expansion is carried out in powers of $\tilde{U}$, with $\lambda_{1}, \lambda_{2}, \lambda_{3}$ determined by the renormalization conditions:

$$
\tilde{\Sigma}(0)=0, \quad \tilde{\Sigma}^{\prime}(0)=0, \quad \tilde{\Gamma}_{\uparrow, \downarrow}(0,0,0,0)=\tilde{U} .
$$

Note that the renormalized form of $\mathcal{L}$ is the same as the original so that the corresponding Hamiltonian is simply a renormalized version of the Anderson model. i.e. 
$\varepsilon_{f} \rightarrow \tilde{\varepsilon}_{f}, \Delta \rightarrow \tilde{\Delta}$ and $U \rightarrow \tilde{U}$. To zero order in $\tilde{U}$ we simply have the result (26) for the spectral density for the renormalized quasi-particles, which when integrated gives the occupation number of the localized state at $T=0$,

$$
n_{\mathrm{f}, \sigma}=\tilde{n}_{\mathrm{f}, \sigma}=\frac{1}{2}-\frac{1}{\pi} \tan ^{-1}\left(\frac{\tilde{\varepsilon}_{\mathrm{f}, \sigma}}{\tilde{\Delta}}\right) .
$$

This result corresponds to the exact formula derived by Langreth ${ }^{41}$, and is a generalization of the Friedel sum rule to the Anderson model with interactions. The corresponding result for the impurity contribution for the specific heat coefficient $\gamma_{\text {imp }}$ is given by

$$
\gamma_{\mathrm{imp}}=\frac{2 \pi^{2}}{3} \tilde{\rho}_{0}(0)=\frac{2 \pi}{3} \frac{\tilde{\Delta}}{\tilde{\epsilon}_{d}^{2}+\tilde{\Delta}^{2}},
$$

which can also be shown to be exact. These results are consistent with Landau Fermi-liquid theory where the quasi-particle number is equal to the number of particles and the specific heat coefficient is determined solely from the contribution of the non-interacting quasi-particles. The non-interacting quasi-particle model must correspond to the fixed point Hamiltonian obtained from the Wilson style of calculation. To first order in $\tilde{U}$ there is a contribution from the direct interaction term between the quasi-particles, but also from the order $\tilde{U}$ terms of the counter terms $\lambda_{1}, \lambda_{2}, \lambda_{3}$, which can be determined from the three renormalization conditions. The results for the impurity contributions to the spin and charge susceptibility are given by

$$
\chi_{\mathrm{imp}}=\frac{\left(g \mu_{\mathrm{B}}\right)^{2}}{2} \tilde{\rho}_{0}(0)\left(1+\tilde{U} \tilde{\rho}_{0}(0)\right), \quad \chi_{\mathrm{c}, \mathrm{imp}}=2 \tilde{\rho}_{0}(0)\left(1-\tilde{U} \tilde{\rho}_{0}(0)\right) .
$$

These results can also be shown to be exact as a consequence of the Ward identity deduced from the conservation of spin and charge. When the direct second order diagram is taken into account, and the corresponding second order contributions from the counter-terms, the renormalized self-energy to order $T^{2}$ can be calculated, an expression for the impurity contribution to the conductivity can be deduced,

$$
\sigma_{\mathrm{imp}}(T)=\sigma_{0}\left\{1+\frac{\pi^{2}}{3}\left(\frac{T}{\tilde{\Delta}}\right)^{2}\left(1+2\left(\frac{\tilde{U}}{\pi \tilde{\Delta}}\right)^{2}\right)+\mathrm{O}\left(T^{4}\right)\right\} .
$$

This result can be shown to correspond to the exact expression derived by Yamada ${ }^{42}$. So, from a simple finite order renormalized perturbation calculation, all the exact results in the low temperature regime for the Anderson model can be derived. These results are, however, in terms of the renormalized parameters of the Anderson model, $\tilde{\varepsilon}_{f}, \tilde{\Delta}$ and $\tilde{U}$. In the Wilson style of calculation, where an explicit renormalization group calculation is performed starting with the 'bare' model in terms $\varepsilon_{f}, \Delta$ and $U$, the renormalized parameters can be deduced by examining the numerical results for the excitations in the neighbourhood of the low temperature fixed point, as was done originally by Wilson for the Kondo model. However, in the localized or Kondo regime of the Anderson model we can exploit the fact that 
there is only one energy scale, the Kondo temperature $T_{\mathrm{K}}$. The relations between the renormalized parameters can be deduced from the condition $n_{d} \rightarrow 1$, and that the charge susceptibility associated with the impurity should vanish in this limit. This implies $\tilde{\varepsilon}_{f} \rightarrow 0$ and $\tilde{U}=\pi \tilde{\Delta}=4 T_{\mathrm{K}}$, so that

$$
\begin{gathered}
\gamma_{\mathrm{imp}}=\frac{\pi^{2}}{6 T_{\mathrm{K}}}, \quad \chi_{\mathrm{imp}}=\frac{\left(g \mu_{\mathrm{B}}\right)^{2}}{4 T_{\mathrm{K}}}, \\
\sigma_{\mathrm{imp}}(T)=\sigma_{0}\left\{1+\frac{\pi^{2}}{16}\left(\frac{T}{T_{\mathrm{K}}}\right)^{2}+\mathrm{O}\left(T^{4}\right)\right\} .
\end{gathered}
$$

and we have all the exact low temperature results in the Kondo regime. As we know that exact solution for the thermodynamic behavior for the symmetric Anderson mode ${ }^{44}$ we can deduce how the renormalized parameters evolve as a function of the bare parameter $U / \pi \Delta$. This is shown in figure 6 , where it can be seen that $\tilde{U} \sim U$ for small values of $U$, and the renormalized scales merge into a single scale for large $U$.

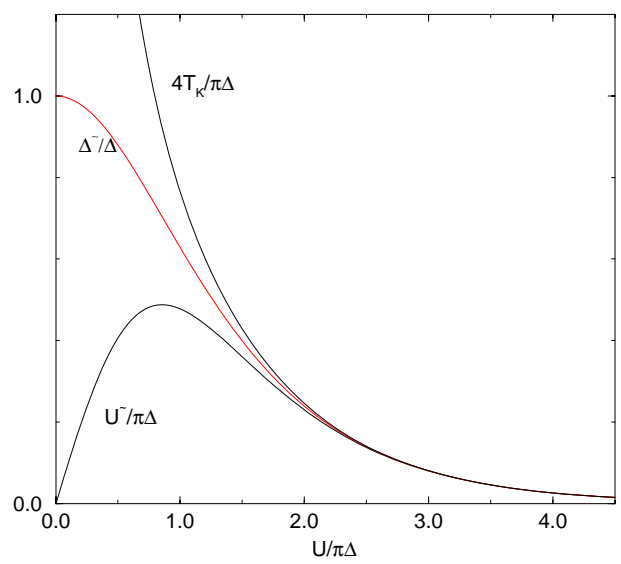

Figure 6. The renormalized parameters, $\tilde{U}$ and $\tilde{\Delta}$ for symmetric impurity Anderson model as a function of the bare parameter ratio $U / \pi \Delta$ taken from reference ${ }^{40}$.

These arguments can be generalized to other impurity models which have a low energy Fermi-liquid fixed point. The corresponding renormalized parameters for the infinite $U N$-fold degenerate Anderson model in the Kondo regime are

$$
\begin{gathered}
\tilde{\Delta}=T_{\mathrm{K}} \frac{N^{2} \sin ^{2}(\pi / N)}{\pi(N-1)}, \quad \tilde{\epsilon}_{d}=T_{\mathrm{K}} \frac{N^{2} \sin (2 \pi / N)}{2 \pi(N-1)}, \\
\tilde{U}=T_{\mathrm{K}}\left(\frac{N}{N-1}\right)^{2},
\end{gathered}
$$


and for the n-channel Anderson Model with $n=2 S$,

$$
\tilde{U}=\pi \tilde{\Delta}=4 T_{\mathrm{K}}, \quad \tilde{J}=-\frac{8}{3} T_{\mathrm{K}},
$$

where $\tilde{J}$ is the renormalized Hund's rule coupling. In all cases $T_{\mathrm{K}}$ is defined such that at $T=0, \chi_{\text {imp }}=\left(g \mu_{\mathrm{B}}\right)^{2} S(S+1) / 3 T_{\mathrm{K}}$.

Nothing has been ignored in the renormalized perturbation approach so that in principle it can be used to look at excitations on any energy scale. It has been set up to be appropriate for the low energy regime and, for the behavior of the model on higher energy scales, the perturbation theory cannot be simply terminated at second order but will involve higher order terms in the expansion in powers of $\tilde{U}$ and the corresponding counter terms. In the Fermi-liquid regime the counter-terms subtract off the ground state expectation values, and the results in this regime can also be obtained by ignoring the counter-terms and simply normal ordering the terms in the renormalized Anderson model with respect to the interacting ground state. The counter-terms play an increasing important role as the energy scale is increased, and reflect the complexity of the interaction terms which are generated in the Wilson approach on intermediate energy scales. As the energy scale is increased the quasi-particles discard their clothing and evolve into the 'bare' or original electrons. As the perturbation expansion up to second order in $\tilde{U}$ gives the leading low temperature results exactly, it would be of interest to see, if taken to third and fourth order in $\tilde{U}$, whether or not it would give the corrections to the Fermi-liquid results exactly. This question is currently being investigated ${ }^{43}$. In the Kondo regime these corrections are known for the magnetization in powers of $\mu_{\mathrm{B}} H / T_{\mathrm{K}}$ from the Bethe ansatz results ${ }^{44,45}$, and for the conductivity up to sixth order in powers of $T / T_{\mathrm{K}}$ from boundary value conformal field theory ${ }^{46}$.

The renormalized perturbation approach has been generalized to the case of an impurity model with a marginal Fermi-liquid fixed point ${ }^{14,21}$, and also to translationally invariant systems ${ }^{47}$. In the latter case, the leading order low temperature results are equivalent to Fermi-liquid theory. The renormalized scattering vertex depends on the wavevectors of the scattered quasi-particles and, as this dependence is not known, it has to be parameterized as in the phemenological Landau Fermiliquid theory; it may be possible to exploit the simplifications that occur in certain limits, such as that of infinite dimensionality.

\section{Renormalization and Model Hamiltonians}

In the last section we saw that a renormalized Anderson model is sufficient to describe the behavior of the original model in the very low energy regime, but that further terms have to be taken into account, either as higher order contributions from the counter-terms in the renormalized expansion, or as extra interaction terms associated with higher order corrections to the low energy fixed point in the Wilson approach. This leads us to the question as to what extent the original Anderson 
model, or similar simplified models, such as the Hubbard model for a lattice system, is a renormalized Hamiltonian, valid only over a limited energy scale. By including the on-site interaction terms only, many other terms involving the Coulomb interaction have been ignored. They may be smaller in magnitude than the on-site term but this does not imply that they can be ignored entirely. We can conjecture that if such terms were to be taken into account explicitly that the results would not be changed significantly; we might just have to modify or renormalize the parameters $\varepsilon_{f}, \Delta$ and $U$ which specify the model. If this is the case, then our original or bare model is in some sense already a renormalized model. We can examine this hypothesis by looking at some of the more obvious terms which have not been included, such as the interactions between the $\mathrm{d}$ - or f-electrons and the conduction electrons, the electron-phonon interactions, or the two-body hybridization or hopping terms.

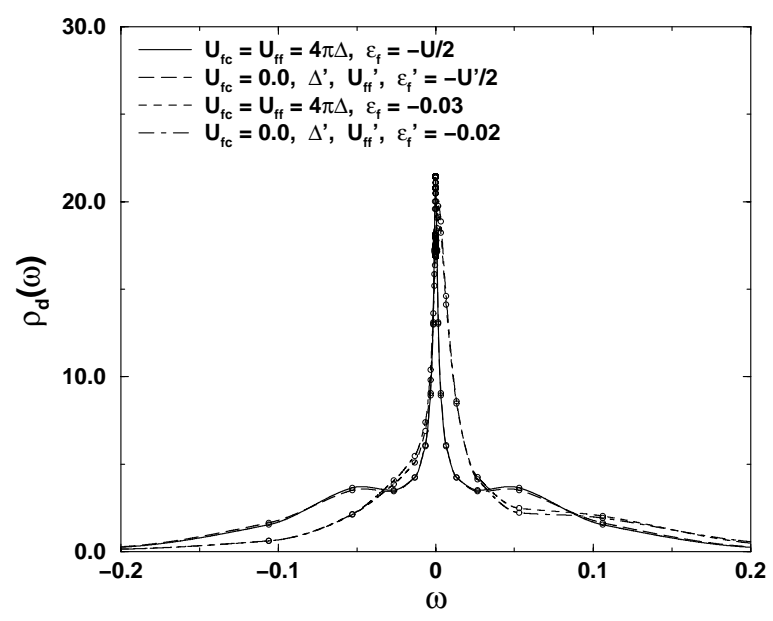

Figure 7. The spectral density $\rho(\omega)$ of the of the local Green's function for the standard Anderson model with an additional Coulomb interaction between the $f$-and conduction electrons, $U_{f c}$, in the local moment and mixed valence regimes with $\Delta=0.01$, and a fitting to the SIAM with the renormalized parameters, $\Delta^{\prime}=0.015, U_{f f}^{\prime}=2.4 \pi \Delta^{\prime}$.

We have made some preliminary studies of the effect of explicitly including a local interaction $U_{c f}$ between the f-electron and the conduction electrons in the Anderson model ${ }^{48}$. In the particle-hole symmetric regime, the results for the felectron spectral density can be reproduced by a standard Anderson model with renormalized parameters $\Delta^{\prime}, \varepsilon_{f}^{\prime}$ and $U_{f f}^{\prime}$. If we change the position of the f-level $\varepsilon_{f}$ in the model with the extra interaction $U_{f c}$ such that we move into the mixed valence regime, it is not obvious that we can reproduce the f-electron spectrum again with the renormalized model (no extra interaction), simply by changing the renormalized level position $\varepsilon_{f}^{\prime}$, without modifying the other parameters. However the results in figure 7 , show that, if we do, we get a fit in both regimes which indicates that we can describe the f-electron behavior on this scale with the simplified model, without 
invoking the $U_{f c}$ interaction explicitly.

However, if we introduce a local coupling to phonons to the SIAM as in the Hubbard-Holstein model (13), then even for $k_{\mathrm{B}} T \ll \omega_{0}$, we find that different renormalized widths have to be used to fit the spectra in the integral valent and mixed valence regimes ${ }^{48}$. It should be of interest to investigate to what extent other terms, such as two-body hybridization terms, can be taken into account simply as a renormalization of the parameters of the standard model and, if so, whether or not these can be changed independently and over what energy scales.

\section{Acknowledgments}

We are grateful for extensive discussions on this subject with T. Costi, A. Oguri, and Y. Shimizu, and to the EPSRC for the support for two of us (ACH, SCB) through research grant (GR/J85349). One of us (RB) was supported by the Deutsche Forschungsgemeinschaft, through the Sonderforschungsbereich 484, and YŌ was supported by the Grant-in-Aid for Scientific Research from the Ministry of Education, Science, Sports and Culture, and also by CREST (Core Research for Evolutional Science and Technology) of Japan Science and Technology Corporation (JST). We also wish to thank the Newton Institute in Cambridge for providing facilities and support for this work during their six month programme on 'Strongly Correlated Electron Systems'.

\section{References}

1. K.G. Wilson, Rev. Mod. Phys. 47, 773 (1975).

2. J. Kondo, Prog. Theor. Phys. 32, 37 (1964).

3. A.C. Hewson, The Kondo Problem to Heavy Fermions (Cambridge Univ. Press, Cambridge, 1993 and 1997).

4. H.R. Krishna-murthy, J.W. Wilkins and K.G. Wilson, Phys. Rev. B 21, 1003 and 1044 (1980).

5. O. Sakai, Y. Shimizu and T. Kasuya, J. Phys. Soc. Jpn. 58, 3666 (1989).

6. T.A. Costi and A.C. Hewson, Phil. Mag. B 65, 1165 (1992); J. Phys.: Condens. Matter 30, L361 (1993); T.A. Costi, A.C. Hewson and V. Zlatić, J. Phys.: Condens. Matter 6, 2519 (1994).

7. P.W. Anderson, Phys. Rev. 41, (1961).

8. W. Hofstetter, Phys. Rev. Lett. 85, 1508 (2000).

9. Y. Shimizu and O. Sakai, J. Phys. Soc. Jpn. 65, 2632 (1996); Y. Shimizu, O. Sakai and S. Suzuki, J. Phys. Soc. Jpn. 67, 3181 (1998).

10. O. Sakai, Y. Shimizu, H. Shiba and K. Satori, J. Phys. Soc. Jpn. 61, 3239 (1992); K. Satori, H. Shiba, O. Sakai and Y. Shimizu, J. Phys. Soc. Jpn. 62, 3181 (1993).

11. K. Chen and C. Jayaprakash, J. Phys.: Condens. Matter 7, L691 (1995); K. Ingersent, Phys. Rev. B. 54, 11936 (1997).

12. R. Bulla, Th. Pruschke and A.C. Hewson, J. Phys.: Condens. Matter 9, 10463 (1997); R. Bulla, M.T. Glossop, D.E. Logan and Th. Pruschke, J. Phys.: Condens. Matter 12, 4899 (2000).

13. H-B Pang and D.L. Cox, Phys. Rev. B 44, 9454 (1991).

14. S.C. Bradley, R. Bulla, A.C. Hewson and G-M Zhang, Eur. Phys. J. B 11, 535 (1999). 
15. R. Bulla and A.C. Hewson, Z. Phys. B 104, 333 (1997).

16. D. Goldhaber-Gordon, J. Göres, M.A. Kastner, H. Shtrikman, D. Mahalu and U. Meirav, Phys. Rev. Lett. 81, 5225 (1998).

17. W. Izumida, O. Sakai and Y. Shimizu, J. Phys. Soc. Jpn. 66, 717 (1997); 67, 2444 (1998)

18. U. Gerland, J. von Delft, T.A. Costi and Y. Oreg, Phys. Rev. Lett. 84, 3710 (2000); D. Boese, W. Hofstetter and H. Schoeller, cond-mat/0010250 (2000).

19. T.A. Costi and C. Kieffer, Phys. Rev. Lett. 76, 501 (1995); T.A. Costi, Phys. Rev. B 55, 3003 (1997); T.A. Costi, Phys. Rev. Lett. 80, 1038 (1998); T.A. Costi and G. Zarand, Phys. Rev. B, 59, 12398 (1999).

20. T.A. Costi, Phys. Rev. Lett. 85, 1504 (2000).

21. R. Bulla, A.C. Hewson and G-M Zhang, Phys. Rev. B 56, 11721 (1997).

22. S.R. White, Phys. Rev. Lett. 69, 2863 (1992); Phys. Rev. B 48, 10345 (1993).

23. Density Matrix Renormalization, ed. I. Peschel et al. (Springer, Berlin, 1999).

24. A. Georges, G. Kotliar, W. Krauth and M.J. Rozenberg, Rev. Mod. Phys. 68, 13 (1996).

25. E. Müller-Hartmann, Z. Phys. B 74, 507 (1989); W. Metzner and D. Vollhardt, Phys. Rev. Lett. 62, 324 (1989); U. Brandt and C. Mielsch, Z. Phys. B 75, 365 (1989); 79, 295 (1990); 82, 37 (1991); A. Georges and G. Kotliar, Phys. Rev. B 45, 6479 (1992); D.M. Edwards, J. Phys.: Condens. Matter 5, 161 (1993).

26. M. Jarrell, Phys. Rev. Lett. 69, 168 (1992).

27. X.Y. Zhang, M.J. Rozenberg and G. Kotliar, Phys. Rev. Lett. 70, 1666 (1993); A. Georges and W. Krauth, Phys. Rev. B 48, 7167 (1993).

28. M. Caffarel and W. Krauth, Phys. Rev. Lett. 72, 1545 (1994).

29. O. Sakai and Y. Kuramoto, Solid State Commun. 89, 307 (1994).

30. R. Bulla, A.C. Hewson and Th. Pruschke, J. Phys.: Condens. Matter 10, 8365 (1998).

31. R. Bulla, Phys. Rev. Lett. 83, 136 (1999).

32. J. Schlipf, M. Jarrell, P.G.J. van Dongen, N. Blümer, S. Kehrein, Th. Pruschke and D. Vollhardt, Phys. Rev. Lett. 82, 4890 (1999).

33. S. Kehrein, Phys. Rev. Lett. 81, 3912 (1998)

34. R. Bulla, T.A. Costi and D. Vollhardt, paper in preparation, (2000).

35. N. Blümer, P.G.J van Dongen and D. Vollhardt, paper in preparation, (2000).

36. R. Bulla, unpublished (1998); R. Bulla and M. Potthoff, Eur. Phys. J. B 13, 257 (2000).

37. Y. Ōno, R. Bulla and A.C. Hewson, cond-mat/0010083 (2000).

38. Th. Pruschke, R. Bulla and M. Jarrell, Phys. Rev. B 61, 12799 (2000); Y. Shimizu, O. Sakai and A.C. Hewson, J. Phys. Soc. Jpn. 69, 1777 (2000); K. Held and R. Bulla, Eur. Phys. J. B 17, 7 (2000).

39. N.N. Bogoliubov and D.V. Shirkov, Introduction to the Theory of Quantized Fields Wiley-Interscience, New York, 1980); L.H. Ryder, Quantum Field Theory (Cambridge Univ. Press, Cambridge, 1985).

40. A.C. Hewson, Phys. Rev. Lett. 70, 4007 (1993).

41. D.C. Langreth, Phys. Rev. 150, 516 (1966).

42. K. Yamada, Prog. in Theor. Phys. 53, 1970 (1975); 54, 316 (1975).

43. A.C. Hewson, paper in course of preparation (2000).

44. A.M. Tsvelik and P.B. Wiegmann, Adv. Phys. 32, 453 (1983).

45. N. Andrei, K. Furuya and J.H. Lowenstein, Rev. Mod. Phys. 55, 331 (1981).

46. F. Lesage and H. Saleur, Phys. Rev. Lett. 82, 4540 (1999).

47. A.C. Hewson, Adv. in Phys. 43, 543 (1994).

48. A.C. Hewson and S.C. Bradley, paper in course of preparation (2000); T.A. Costi and A.C. Hewson, J. Mag. Mag. Mat. 108, 129 (1992). 